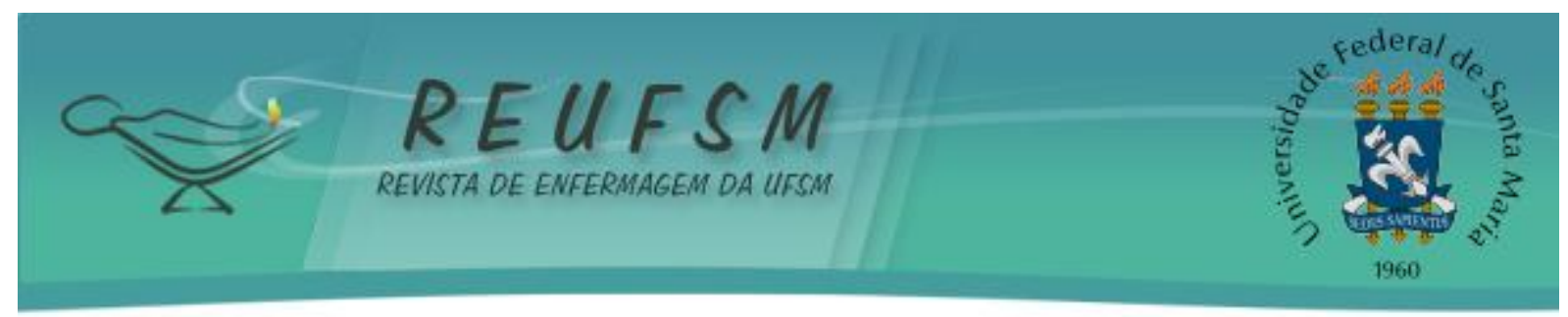

REVISÃO

\title{
TENDÊNCIAS HISTÓRICAS NO CONHECIMENTO DA ENFERMAGEM E O SISTEMA ÚNICO DE SAÚDE
}

\section{HISTORICAL TENDENCIES IN NURSING KNOWLEDGE AND THE SINGLE HEALTH \\ TENDENCIAS HISTÓRICAS EN EL CONOCIMIENTO DE ENFERMERÍA Y EL SISTEMA ÚNICO DE SALUD}

\author{
Sueli Goi Barrios ${ }^{1}$ \\ Adelina Giacomelli Prochnow ${ }^{2}$ \\ Maria Denise Schimith ${ }^{3}$ \\ Ana Cristina Passarella Brêtas ${ }^{4}$
}

RESUMO: O objetivo deste estudo foi analisar as tendências históricas nas produções científicas da enfermagem relacionadas ao Sistema Único de Saúde, no período de 1990 a 2006. A pesquisa bibliográfica foi realizada através dos resumos das dissertações e teses encontradas nos Catálogos do Centro de Estudos e Pesquisa em Enfermagem, selecionando-se 31 resumos que continham Sistema Único de Saúde como descritor. Os temas foram agrupados em cinco categorias: Atuação do Profissional Enfermeiro; Controle Social; Formação Profissional em Saúde; Organização dos Serviços de Saúde e Direito à Saúde. Concluiu-se que a tendência na construção do conhecimento de enfermagem vincula-se aos aspectos técnicos e operacionais dos enfermeiros, em detrimento das abordagens relacionadas às questões sociais e políticas do sistema de saúde, demonstrando um distanciamento entre o movimento para consolidação do SUS e as produções acadêmicas da enfermagem.

Descritores: Enfermagem; Sistema Único de Saúde; Gestão em saúde.

ABSTRACT: The purpose of this study was to identify and analyze historical tendencies in the construction of Nursing knowledge, from 1990 to 2006. A bibliographic research was performed through the dissertations and theses found in the catalogue of the Center for Studies and Research in Nursing. Thirty one (31) abstracts that contained the Single Health System as a descriptor were selected. The subjects were grouped into five categories: performance of professional nurses, social control, health professional training, organization of health services and health rights. It was concluded that the tendencies in the construction of Nursing knowledge are linked to the technical and operational aspects of the nurses, over the approach related to social and political issues of the health system, showing a gap throughout the movement to consolidate the SUS and academic productions of Nursing.

Descriptors: Nursing; Single Health System; Health management.

RESUMEN: El objetivo del estudio que se ha planteado es analizar las tendencias históricas en las producciones científicas de enfermería relacionadas al Sistema Único de Saúde, en el período de 1990 a 2006, encontradas en los catálogos del Centro de Estudio e

\footnotetext{
${ }^{1}$ Mestre em Enfermagem pela Universidade Federal de Santa Maria. Enfermeira do Centro Regional de Saúde do Trabalhador. sueligbarrios@hotmail.com

2 Doutora em Enfermagem pela Universidade Federal do Rio de Janeiro. Professora Adjunta da Universidade Federal de Santa Maria - RS. agp.sma@terra.com.br

3 Mestre em enfermagem pela Universidade Federal do Rio Grande do Sul. Professora Assistente da Universidade Federal de Santa Maria - RS. ma.denise@yahoo.com.br

${ }^{4}$ Doutora em Enfermagem pela Universidade Federal de São Paulo. Professora Associada da Universidade Federal de São Paulo. acpacpbretas@unifesp.br
} 




Investigación en Enfermería. La investigación bibliográfica fue realizada a través de los resúmenes de las tesis de maestría y doctorado encontradas en los Catálogos do Centro de Estudos e Pesquisas em Enfermagem. Se seleccionaron 31 resúmenes, los cuales aportaban Sistema Único de Saúde como palabra clave. Se agruparon los temas en cinco categorías: actuación del profesional enfermero, control social, formación profesional en salud, organización de los servicios de salud y derecho a la salud. Se ha concluido que la tendencia en la construcción del conocimiento de enfermería se aúna a los aspectos técnicos y operacionales de los enfermeros, en detrimento de los abordajes asociados a las cuestiones sociales y políticas del sistema de salud, demostrando un distanciamiento entre el movimiento para la consolidación del SUS y las producciones académicas de enfermería.

Descriptores: Enfermería; Sistema Único de Saúde; Gestión en salud.

\section{INTRODUÇÃO}

A Constituição Federal de 1988, além de garantir a saúde como direito de todos e dever do Estado, refere-se ao acesso universal e igualitário de todos os cidadãos por meio de ações e serviços de saúde integrados em uma rede regionalizada e hierarquizada, organizada de acordo com as diretrizes de: descentralização, atendimento integral e participação da comunidade. ${ }^{1}$ Dois anos depois, a Lei Orgânica da Saúde, Lei n ${ }^{\circ}$ 8080/90, traz como responsabilidade do SUS o acesso da população aos serviços de saúde em todos os níveis de complexidade, de acordo com cada caso. ${ }^{2}$

A motivação para realizar este estudo decorre da experiência das autoras na gestão regional do SUS, no Estado do Rio Grande do Sul, na qual pode ser observada a grande participação de enfermeiros, seja assumindo o cargo de Secretário Municipal de Saúde, seja atuando em sua assessoria. E também o fato da produção científica da enfermagem brasileira ter seu incremento a partir de 1990, coincidindo com a implantação do SUS. Desde então, o processo de cientifização consolidou grupos de pesquisa, formando pesquisadores e lutando por seus espaços. ${ }^{3}$

Portanto, estudar a produção de conhecimento da enfermagem acerca do SUS torna-se relevante, à medida que pretende contribuir com a compreensão dos aspectos científicos pesquisados pela profissão que, de alguma forma, tem participado na construção dessa política.

Nesse sentido, este estudo, ao examinar a produção de conhecimento da enfermagem, questiona: Que aspectos do SUS estão sendo pesquisados pela enfermagem? Qual é o volume de produção apresentado? A produção de conhecimento da enfermagem acompanhou os movimentos de consolidação da política do SUS? No caminho da elaboração desta pesquisa, admitia-se que a produção teórica no tema revelasse a prática profissional.

Assim, elaborou-se como objetivo analisar as tendências históricas nas produções científicas da enfermagem (dissertações e teses) relacionadas ao SUS, no período de 1988 a 2006, encontrados nos catálogos do Centro de Estudo e Pesquisa em Enfermagem (CEPEn $-\mathrm{ABEN})$.

\section{MÉTODO}

A pesquisa configurou-se como bibliográfica, constituída pelos resumos das teses e dissertações encontradas nos catálogos do Centro de Estudo e Pesquisa em Enfermagem (CEPEn), disponíveis no site da Biblioteca Virtual da Enfermagem, bem como as 


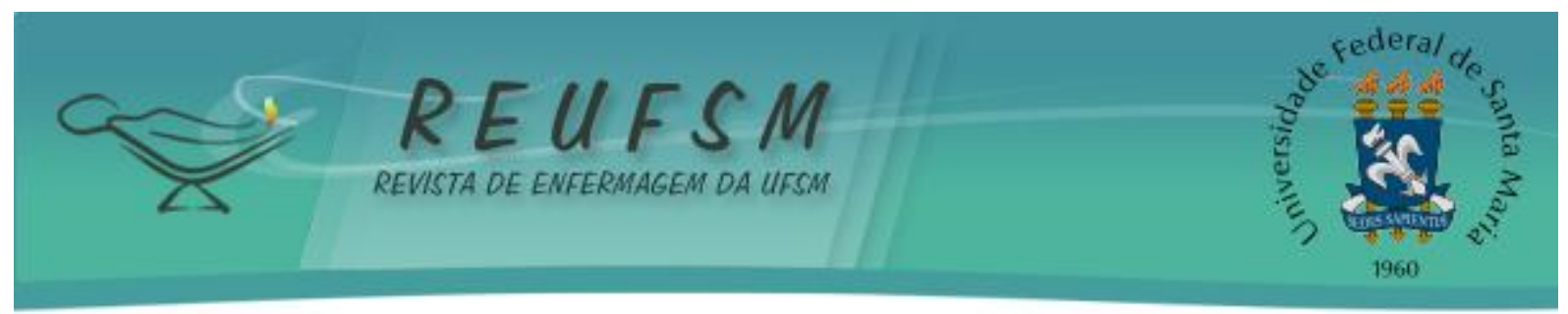

informações armazenadas em CD-ROM elaborado pela Associação Brasileira de Enfermagem (ABEn), correspondentes aos anos de 1979 a 2006.

O período pesquisado foi a partir de 1990, respeitando o marco temporal do da publicação da Lei 8.080/90, que regulamenta o SUS, estendendo-se até 2006, por ser este o último ano de compilação disponível no momento da pesquisa. A busca foi realizada durante os meses de setembro e outubro de 2008. Justifica-se a opção pelo CEPEn, por tratar-se do maior banco de dados que congrega as dissertações e teses dos enfermeiros.

A busca foi realizada através do descritor Sistema Único de Saúde, resultando em 31 trabalhos, ou seja, todos cujos resumos que continham esse descritor, representados por oito teses e 23 dissertações de mestrado.

Para a análise dos dados, utilizou-se a análise temática, que consiste em agregar em "núcleos de sentido" os resultados que representem algo para o objeto pesquisado. 4:316 Seguiram-se as três etapas da análise temática: a pré-análise; a exploração do material, buscando encontrar as categorias; e a classificação e agregação dos dados.

Os aspectos do SUS que a enfermagem tem se dedicado a pesquisar tratam do trabalho da enfermagem e da equipe de saúde, da organização formal de serviços de saúde, do controle social, em especial os espaços formais estabelecidos em lei, da concepção de saúde como direito de cidadania e da formação profissional para atuação no sistema de saúde. A partir disso, os dados foram agrupados nos seguintes núcleos de sentido: Atuação do Profissional Enfermeiro; Controle Social; Organização de Serviços de Saúde; Formação Profissional e Direito à Saúde.

\section{ANÁLISE E DISCUSSÃO DOS RESULTADOS}

\section{A distribuição temporal das produções encontradas está no Gráfico 1.}

Gráfico 1: Produções de enfermagem com descritor SUS no período de 1990-2006

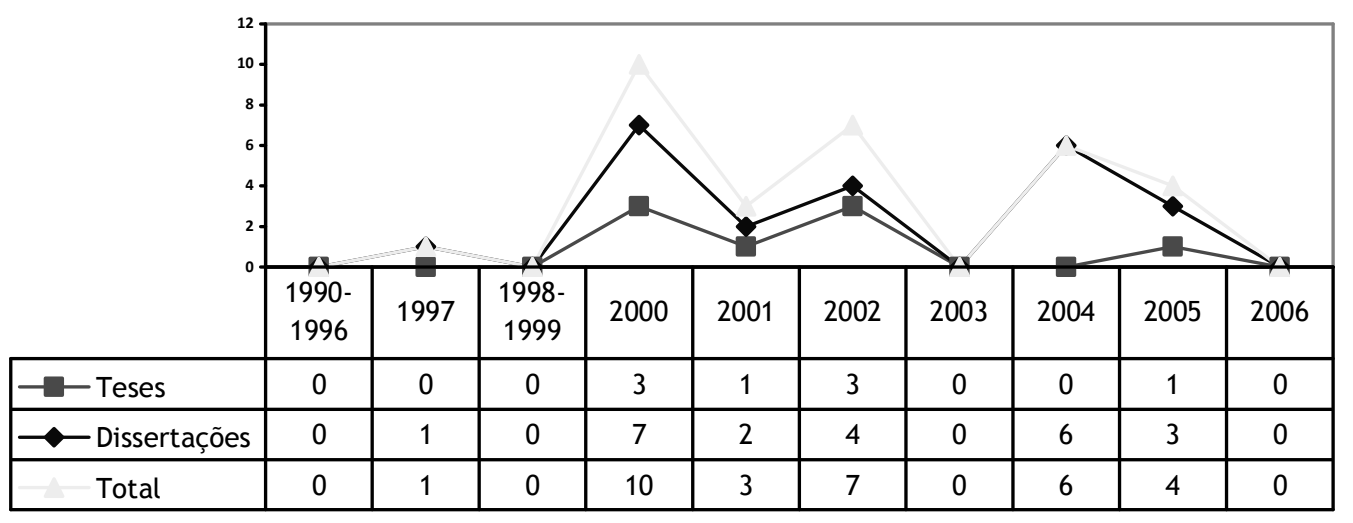

Fonte: Catálogo do CEPEn

Ressalta-se a lacuna apresentada entre 1990 e 1999, quando apenas uma dissertação foi realizada, indicando que, durante toda essa década, o SUS não foi pesquisado por enfermeiros. 0 mesmo ocorre em 2003 e 2006, embora nesses anos tenham ocorrido processos importantes no país, com repercussões significativas para o sistema de saúde, como: mudança do governo federal, que se apresentava com discurso de fortalecimento do SUS; implantação de polos de educação permanente, com fortalecimento do diálogo e aproximação entre instituições formadoras e de trabalho; ${ }^{5}$ ampliação do Programa de Saúde da Família; ${ }^{6}$ construção do pacto pela saúde, dentre 


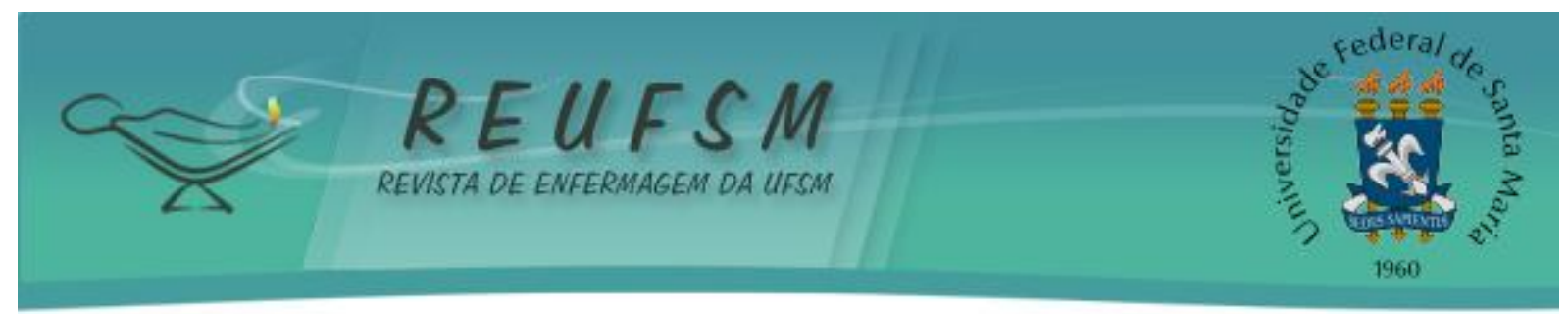

outros. $^{7}$ Pode-se afirmar que a produção de conhecimento da enfermagem não acompanhou, nos anos citado, os movimentos de consolidação da política do SUS, muito embora os processos mencionados tenham ampliado os espaços de participação do profissional enfermeiro.

A maior contribuição da construção do conhecimento acerca desta temática surge em São Paulo (Universidade de São Paulo) e de Santa Catarina (Universidade Federal de Santa Catarina), confirmando a tendência presente ainda hoje, em decorrência de que as pesquisas encontram-se concentradas nesses estados, os quais detêm o maior número de programas de pós-graduação. ${ }^{3}$

\section{Atuação do profissional enfermeiro}

Entre os temas mais abordados estão os referentes às atividades desenvolvidas pelos enfermeiros junto às Unidades Básicas de Saúde (UBSs). Os enfermeiros têm atuado em atividades de apoio e não em atividades decisórias, evidenciando uma deficiência na gestão do trabalho em saúde, o que acarreta uma prática desarticulada e não comprometida. $^{8}$

Corroborando com o achado acima, pesquisas apontam a fragilidade dos enfermeiros em assumir funções decisórias no SUS, relacionada com um "vazio" político, o qual compromete a sua participação em espaços públicos de disputas de poder. ${ }^{9}$ Esse fato pode estar relacionado com a falta de conhecimento no campo da política com implicação especialmente sobre a política de saúde e de gestão do SUS.

Nesse sentido, um resumo analisado afirma que o SUS ampliou o espaço de trabalho do enfermeiro, no entanto, o trabalho é individualizado, não há trabalho em equipe, o que compromete a integralidade da atenção. ${ }^{10}$ Outro estudo evidencia que há dicotomia entre a assistência e a gerência, o que também ameaça a integralidade. ${ }^{11}$

Entende-se como necessário que os enfermeiros se reconheçam como sujeitos históricos, pois, a depender da coerência de suas posições, poderão contribuir em maior ou menor intensidade para construção do SUS.

As pesquisas analisadas nesse núcleo de sentido apontam para a configuração de um modelo com forte predomínio para atendimento individual, fragmentado, curativo, sem planejamento, com pouca adesão ao sistema de saúde, pouca participação política e social, reproduzindo o modelo hegemônico ainda predominante. Analisa-se que isso pode ser decorrente da fragilidade no processo de formação, o qual discute o SUS de forma descontextualizada social e politicamente.

\section{Controle social}

A respeito do significado da participação dos profissionais de saúde no Conselho Municipal de Saúde, um resumo analisado identificou que alguns profissionais de saúde assimilam a política municipal, assumindo a proposta do gestor sem questioná-la, fato que interfere no processo de democratização da saúde. ${ }^{12}$

Destaca-se o estudo que objetivou compreender a percepção de controle social e o processo de construção da participação popular na política pública de saúde no Município de Chapecó/SC, cujos resultados apontam para avanços no trabalho realizado pelo Conselho Local de Saúde, garantido através da participação de conselheiros em espaços de discussão da política pública do município. ${ }^{13}$

Outro estudo revela que a percepção de ser conselheiro está mais ligada à ideia de assistencialismo do que de transformação social. A participação da comunidade é incipiente, o que perpetua a representação e caracteriza a vacância do cargo. ${ }^{14}$ 




Ressalta-se a importância da participação do usuário no âmbito do SUS, demonstrando possibilidades desse segmento contribuir para a formulação das políticas públicas de saúde. Para que isso ocorra, entende-se como necessário a reinvenção e ressignificação deste espaço de participação popular. Essa ressignificação passa pelo reconhecimento do protagonismo da população, para além dos aspectos previstos nas normas e nas leis.

\section{Formação profissional}

Apenas dois pesquisadores tomaram a formação profissional para o SUS como objeto central de suas pesquisas. Um estudo avaliou as escolas médicas e a formação deste profissional para o SUS. ${ }^{15}$ Outro, ao estudar a formação do enfermeiro, identificou fragilidade nas discussões que promovam a integralidade e participação dos acadêmicos no processo ensino-aprendizagem. ${ }^{16}$

Observou-se que a construção de conhecimento da enfermagem no campo da formação profissional apontou uma distância ainda significativa entre as necessidades da população usuária do sistema de saúde e a formação, transformando-se em obstáculo para a efetivação de mudanças no atual modelo de atenção à saúde.

Nesse sentido, é importante reconhecer que há cisão entre ensino e trabalho. São comuns os locais de inserção dos alunos permanecerem nos limites de cada instituição de forma pontual, onde os serviços do SUS servem apenas como cenário de prática para o ensino, não ocorrendo integração entre a universidade e a gestão, de maneira que juntos repensem a lógica dos processos pedagógicos, de trabalho e de gestão, resultando na transformação das práticas institucionais de ensino/serviço. ${ }^{17}$

Isso aponta para necessidade de repensar as instituições formadoras, de modo que percebam a importância em buscar maior aproximação à realidade do sistema público de saúde do país, ao mesmo tempo em que é imprescindível que o SUS assuma sua responsabilidade legal de ordenar a formação de recursos humanos para área de saúde.

\section{Organização de serviços de saúde}

Os trabalhos encontrados estão focados nos serviços de referência e contrarreferência. Ao investigar um Distrito Sanitário do Município de São Paulo, um estudo constatou que existem mecanismos formais e informais de referenciar e contrarreferenciar, no entanto estes, por si sós, não garantem acesso aos diferentes serviços de saúde. ${ }^{18}$ Esse achado foi confirmado por outro estudo, inferindo que a dificuldade de acesso, apesar da existência do sistema de referência e contrarreferência, se mantém tanto em UBSs quanto em unidades de maior densidade tecnológica, não garantindo a integralidade da atenção. ${ }^{19}$

Tem-se utilizado historicamente, entre os serviços de saúde, o que se convencionou chamar de referência e contrarreferência, uma normativa que expressa a "cultura do encaminhamento", retratando as antigas formas de gestão do cuidado capturados por normas, desconsiderando as singularidades e serializando as práticas. ${ }^{20: 463}$

Corroborando com isso, uma pesquisa que buscou compreender o envolvimento dos trabalhadores nas propostas de organização de serviços de saúde identificou um modelo de gestão verticalizado e excludente, que dificulta a implicação do trabalhador no processo de trabalho. ${ }^{21}$

Percebe-se, portanto, a centralidade de atos normativos e gestão não participativa, o que predispõe à alienação dos trabalhadores e à dicotomia entre gestão e atenção. Os estudos confirmam a dificuldade de acesso à rede de ações e serviços, que vai 


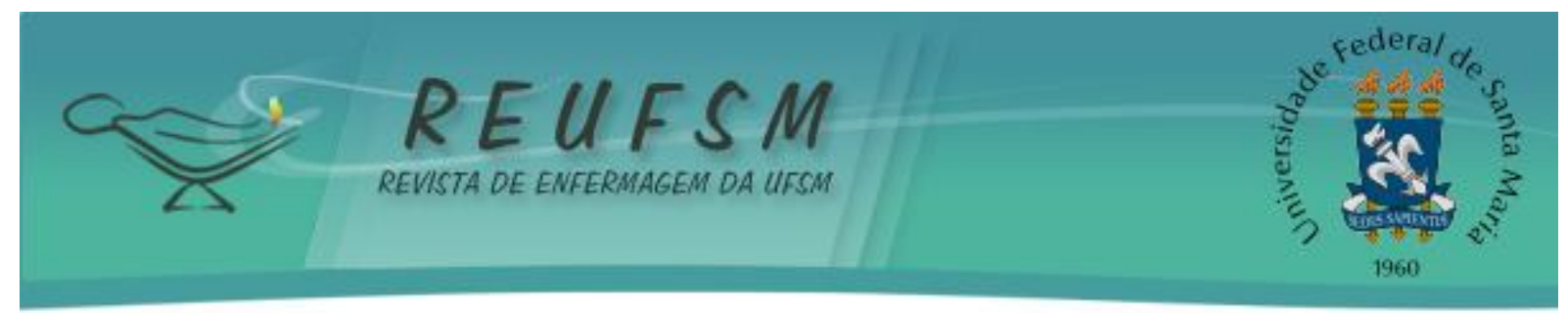

desde a atenção básica até os serviços de maior densidade tecnológica, o que se evidencia no cotidiano dos serviços do SUS.

\section{Direito à saúde}

Os estudos realizados pela enfermagem revelam obstáculos na sedimentação do direito à saúde. Destaca-se o estudo que afirma que o direito à saúde não está garantido na reforma do setor saúde. Há uma ameaça de privatização do sistema, representada tanto pelo Programa de Agentes Comunitários da Saúde (PACS) e Programa Saúde da Família (PSF), quanto pela transformação de estruturas de maior complexidade em organizações não estatais, submetendo a assistência à saúde às leis de mercado. ${ }^{22}$

As pesquisas identificaram também conflitos e contradições no processo de construção do SUS na região semiárida do sertão nordestino brasileiro, onde a política democrática do SUS não encontra eco, devido à política coronelista e oligárquica que defende $a$ indústria de favores em troca de votos de permanência no poder. ${ }^{23}$

Soma-se a isso o estudo sobre a concepção dos enfermeiros da Estratégia Saúde da Família em relação aos princípios do SUS. Nele conclui-se que, na maioria das vezes, os enfermeiros os relatam e os compreendem apenas de forma memorizada, com pouca clareza de seus significados. ${ }^{24}$

O SUS necessita, portanto, retomar o movimento de Reforma Sanitária, na perspectiva de garantir, no cotidiano, a saúde como direito de cidadania.

Para tanto, entende-se como necessário que os defensores do SUS discutam alternativas de gestão que possibilitem a superação dos obstáculos construídos pelos contrários, ao mesmo tempo em que a sociedade deve exigir do Estado brasileiro responsabilidade social para com a saúde e a qualidade de vida dos cidadãos.

Os núcleos de sentido aqui analisados são coerentes entre si e se interrelacionam, demonstrando que as tendências históricas da construção do conhecimento da enfermagem apontam para a necessidade de se ter profissionais críticos e competentes para atuar no SUS. A formação deveria estar imbricada com realidade, tornando indissociável a relação entre a teoria e a prática. Para tanto, é importante que os docentes tomem consciência da necessidade de instrumentalizar técnica, científica e politicamente os futuros profissionais frente às questões sociais e políticas do país, em especial as relacionadas com o setor saúde. ${ }^{25}$

\section{CONSIDERAÇÕES FINAIS}

Este estudo possibilitou a imersão no campo da investigação científica da enfermagem, permitindo conhecer a produção existente sobre o tema SUS, desde a sua criação. Um aspecto verificado foi a tendência da enfermagem em dedicar-se à realização de pesquisas sobre controle social, assistência de enfermagem, integralidade da atenção e formação profissional, com maior vinculação aos aspectos técnicos e operacionais do cotidiano dos enfermeiros.

Porém, no campo da gestão pública que requer a inserção social e política do enfermeiro em espaços de construção e organização de sistemas de saúde, está aquém do desejado, apresentando-se como um campo pouco explorado, conforme revelam os dados desta pesquisa.

Não se pode dizer que a enfermagem brasileira não esteja inserida no processo de construção do SUS, entretanto parece haver um distanciamento entre o movimento nacional de luta pela reforma sanitária e consolidação do SUS e as produções acadêmicas da enfermagem publicadas no BDENF, cujas abordagens não têm contribuído 


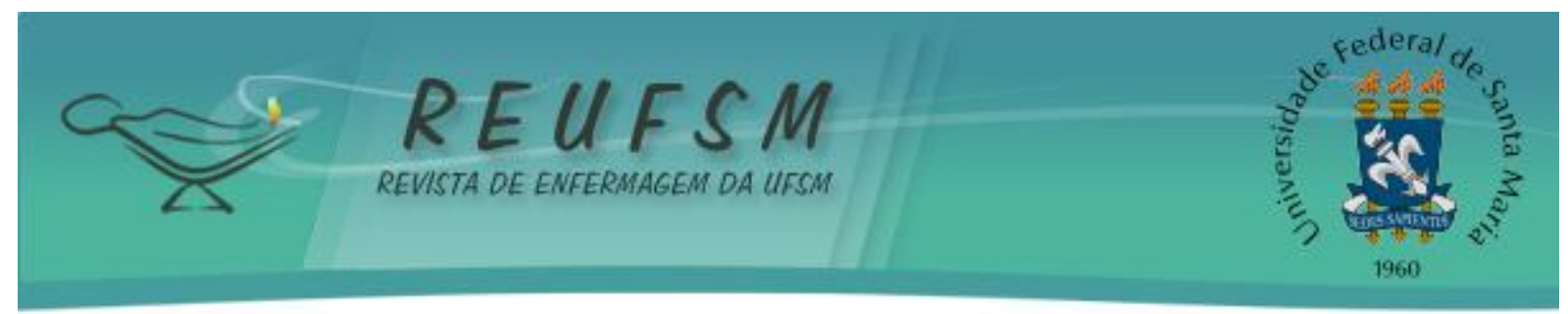

significativamente com as mudanças no modelo de atenção e formação profissional, com potência para afirmar o SUS como política pública de inclusão social.

Este artigo possui alguns limites, dentre os quais se destaca a fonte de busca dos trabalhos, pois, incluindo outras, poder-se-ia abranger maior número de produções. Embora sendo considerado o maior banco de teses e dissertação da enfermagem, muitas não se encontram nesse catálogo, o que impediu a análise ampla da inserção dos pesquisadores da enfermagem e sua produção do conhecimento sobre o SUS.

Todavia, é de fundamental importância que a enfermagem se dedique à construção de conhecimento científico, integrando ensino e trabalho, aprofundando seus estudos a respeito do SUS. O que significa que um dos desafios postos aos pesquisadores de enfermagem é o de atender às prioridades de pesquisa da Agenda Nacional do Ministério da Saúde, as quais apontam para realização de estudos cujos resultados possam melhorar os serviços e ações de saúde prestados no âmbito do SUS, com potencialidade para contribuir com mudanças que impactem positivamente na melhoria dos níveis de saúde e na qualidade de vida da população brasileira.

\section{REFERÊNCIAS}

1. Brasil. Constituição Brasileira. Brasília: Congresso Nacional; 1988.

2. Brasil. Lei $n^{\circ}$. 8080, de 19 de setembro de 1990. Dispõe sobre as condições para a promoção, proteção e recuperação da saúde, a organização e o funcionamento dos serviços correspondentes e dá outras providências. Diário Oficial da União. 20 set.1990. Seção I.p.18.055.

3. Salles EB, Barreira IA. Formação da comunidade científica de enfermagem no Brasil. Texto \& contexto enferm [periódico na Internet]. 2010 mar [acesso em 2010 Dez 12]; 19(1):137-46. Disponível em: http://www.scielo.br/pdf/tce/v19n1/v19n1a16.pdf

4. Minayo MCS. O desafio do conhecimento: pesquisa qualitativa em saúde. $11^{\mathrm{a}}$ ed. São Paulo: Ed. Hucitec; 2008.

5. Ministério da Saúde (BR). Portaria 198 de 13 de fevereiro de 2004. Política Nacional de Educação Permanente em Saúde. Brasília: Ministério da Saúde; 2004.

6. Ministério da Saúde (BR). Secretaria de atenção a Saúde. Departamento de atenção Básica. Coordenação de acompanhamento e avaliação da atenção Básica. Informe da Atenção Básica. O Projeto de Expansão e Consolidação da Saúde da Família - PROESF. Ano IV, jul-ago; 2003.

7. Ministério da Saúde (BR). Portaria n. 399/GM DE 22 de fevereiro de 2006. Divulga o Pacto pela Saúde 2006 - Consolidação do SUS e aprova as Diretrizes Operacionais do Referido Pacto [Internet]. 2006 [acesso em 2009 set 20]. Disponível em: http://dtr2001.saude.gov.br/sas/PORTARIAS/Port2006/GM/GM-399.htm

8. Cheade MFM. Caracterização das atividades dos enfermeiros nas secretarias municipais de saúde do Estado de Mato Grosso do Sul [dissertação de mestrado].São Paulo: Escola Paulista de Medicina, 2000.

9. Melo CMM, Santos TA. A participação política de enfermeiras na gestão do Sistema Único de Saúde em nível municipal. Texto \& contexto enferm [periódico na internet]. 2007 set [acesso em 2010 out 26]; 16(3):426-32. Disponível em: http://www.scielo.br/pdf/a07v16n3.pdf 


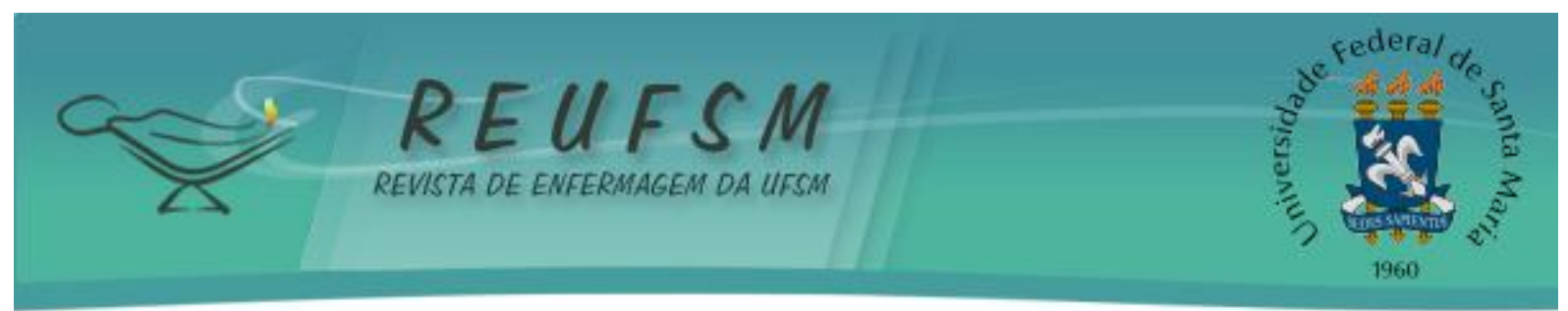

10. Thumé E. Práticas dos enfermeiros na Atenção Básica em Saúde na região sul do Rio Grande do Sul [dissertação de mestrado]. Florianópolis: Universidade Federal de Santa Catarina, 2000.

11. Filgueiras SRD. Atuação do enfermeiro na Estratégia de Saúde da Família: o despertar de um novo cenário no Município de Volta Redonda/RJ [dissertação de mestrado]. Rio de Janeiro: Universidade Federal do Estado do Rio de Janeiro, 2005. 12. Coêlho AA. Significado da atuação de profissionais no Conselho Municipal de Saúde [dissertação de mestrado]. João Pessoa: Universidade Federal da Paraíba, 2001.

13. Silva MT. Controle social e participação popular na política de saúde: ação/intervenção de enfermagem junto a um Conselho Local de Saúde, Chapecó-SC [dissertação de mestrado]. Florianópolis: Universidade Federal de Santa Catarina, 2004.

14. Rettori ECS. Participação da comunidade: um estudo com conselheiros de saúde em São José dos Campos [dissertação de mestrado]. São Paulo: Universidade Federal de São Paulo, 2004.

15. Amaral JL. Avaliação e transformação das escolas médicas: uma experiência, nos anos 90, na ordenação de recursos humanos para o SUS [dissertação de mestrado]. Rio de Janeiro: Universidade do Estado do Rio de Janeiro, 2002.

16. Müller AR. Reflexão crítica acerca do Sistema Único de Saúde - SUS na formação profissional: ponto de vista de acadêmicos de enfermagem [dissertação de mestrado]. Florianópolis: Universidade Federal de Santa Catarina, 2002.

17. Guizardi FL, Stelet B, Pinheiro R, Ceccin RB. A formação de profissionais orientada para integralidade e as relações político-institucionais na saúde: uma discussão sobre a interação ensino-serviço. In: Pinheiro R, Ceccim RB, Mattos RA (org). Ensinar Saúde: a integralidade e o SUS nos cursos de graduação na área da saúde. Rio de Janeiro: CEPESQ: ABRASCO; 2006.

18. Maeda ST. Gestão da referência e contra referência na atenção ao ciclo gravídicopuerperal: a realidade do Distrito de Saúde do Butantã [tese de doutorado]. São Paulo: Universidade Federal de São Paulo, 2002.

19. Soito RXS. Sistema Único de Saúde: da teoria à prática da integralidade [dissertação de mestrado]. São Paulo: Universidade de São Paulo, 2004.

20. Franco TB. As redes na micropolítica do processo de trabalho em saúde. In: Pinheiro R, Mattos RA (org). Gestão em redes: práticas de avaliação, formação e participação na saúde. Rio de Janeiro: CEPESC; 2006.

21. Cunha BFG. Implicação dos trabalhadores de ambulatórios municipais, em UberabaMG, na reorganização de serviços preconizada pelo Sistema Único de Saúde [dissertação de mestrado]. Belo Horizonte: Universidade Federal de Minas Gerais, 2004.

22. Calipo SM. Saúde, Estado e ética - NOB/96 e lei das organizações sociais: a privatização da instituição pública na saúde [dissertação de mestrado]. São Paulo: Universidade de São Paulo, 2002.

23. Bosco Filho J. Avançando em direção ao passado: a luta pela construção/consolidação do SUS em Mossoró/RN [dissertação de mestrado]. João Pessoa: Universidade Federal da Paraíba, 2002. 




24. Bernardes EH. Princípios do Sistema Único de Saúde: concepção dos enfermeiros da estratégia de saúde da família, de uma cidade do Estado de Minas Gerais [dissertação de mestrado]. Ribeirão Preto: Universidade de São Paulo, 2005.

25. Canônico RP, Brêtas ACP. Significado do Programa Vivência e Estágios na Realidade do Sistema Único de Saúde para formação profissional na área de saúde. Acta paul enferm [periódico na internet]. 2008 [acesso em 2010 out 26]; 21(2):256-61. Disponível em: http://www.scielo.br/pdf/ape/v21n2/a04v21n2.pdf

Data de recebimento: $27 / 10 / 2010$

Data de aceite: $20 / 12 / 2011$

Contato com autora responsável: Sueli Goi Barrios

E-mail: sueligbarrios@hotmail.com 